\title{
Efficient Resource Allocation and Sectorization for Fractional Frequency Reuse (FFR) in LTE Femtocell Systems
}

\author{
Mohammad T. KAWSER, Mohammad R. ISLAM, Kazi I. AHMED, Mir R. KARIM, Jaeed B. SAIF
}

Dept. of Electrical and Electronic Engineering, Islamic University of Technology, Board Bazar, Gazipur-1704, Bangladesh

mkawser@hotmail.com, rakibultowhid@yahoo.com, \{ishfaq06,mrejaul, saifsiam\}@iut-dhaka.edu

\begin{abstract}
The Fractional Frequency Reuse (FFR) is a resource allocation technique that can effectively mitigate inter-cell interference (ICI) in LTE based HetNets and it is a promising solution. Various FFR schemes have been suggested to address the challenge of interference in femtocell systems. In this paper, we study the scopes of interference mitigation and capacity improvement. We propose a resource allocation scheme that gradually varies frequency resource share with distance from the eNodeB for both macrocells and femtocells in order to attain better utilization of the resources. This is performed effectively using three layers in the cell. The proposal also employs high number sectors in a cell, low interference and good frequency reuse. Monte-Carlo simulations are performed, which show that the proposed scheme achieves significantly better throughput compared to the existing FFR schemes.
\end{abstract}

\section{Keywords}

LTE, Fractional Frequency Reuse (FFR), femtocells, resource allocation

\section{Introduction}

Long term evolution (LTE), based on 3GPP standards, is the latest step in the evolution of cellular services. The ever increasing demand for higher data rates and better quality of services has motivated the consideration of heterogeneous networks (HetNets) in LTE. We consider HetNets that comprise macrocells and femtocells. The femtocells provide high quality indoor communications with low transmit power and it improves the spectral efficiency at lower cost [1], [2]. The femtocell base station or Home eNodeB (HeNB) can be operated in either open or closed access mode. In open access mode, the HeNB provides services to all subscribers. On the other hand, in closed access mode, the HeNB controls the access of users and provides services only to the users who belong to a closed subscriber group (CSG).

Like most literature addressing the interference issues in femtocell systems, we assume that a good number of femtocells are deployed where significant macro coverage already exists. Since femtocells use the same spectrum as do the macrocells, the co-channel interference (CCI) between macrocells and femtocells must be tolerable [3]. Apart from the CCI among neighboring macrocells, there are, in fact, two types of interference concerns in femtocell systems: co-tier interference, which occurs between neighboring femtocells and cross-tier interference, which occurs between femtocells and macrocells. These interferences can arise in six possible scenarios as shown below.

1. Femto DL is affected by macro eNB.

2. Femto UL is affected by macro-users.

3. Macro DL is affected by HeNB.

4. Macro UL is affected by femto-users.

5. Femto DL is affected by another HeNB

6. Femto UL is affected by femto-users.

Scenario \#1 can have substantial interference because macro eNB typically uses much higher power than HeNB. Scenarios \#2 and \#3 can have substantial interference when the macro-users stay close to the HeNB. Scenario \#4 offers substantial interference when there are a large number of femtocells deployed. Then the aggregate UL interference from the large number of femto-users becomes high. Scenarios \#5 and \#6 exhibit substantial interference when the femtocells are randomly located, which is more likely in residential deployments. In public or enterprise deployments, femtocells may be carefully located at predesigned places limiting the interference [4].

To mitigate the interference in femtocell systems, various inter-cell interference coordination (ICIC) techniques have been suggested. One of the major ICIC techniques is fractional frequency reuse (FFR), which also yields better frequency reuse [5-8]. In case of FFR, the cell is divided into different regions and the available frequency band is also divided into several sub-bands. Then different sub-bands are allocated to different regions. Also, different power levels may be used for different sub-bands in different regions. A FFR deployment mitigating interference in femtocell systems successfully can achieve high spectral efficiency. To address the interference issues effectively, a number of FFR deployment schemes have been suggested. The strict FFR [7], soft FFR [9], FFR-3 [6], [10], optimal static FFR (OSFFR) [11] and 3-layer/3-sector FFR 
[12] are examples of such schemes and they employ different methods in splitting the cells, splitting the available bandwidth and allocating different resources to different regions. [13] uses graph theory and [14] uses two-stage heuristic approach to find optimal design of FFR deployment.

It may be noted that the co-tier and cross-tier interferences arise because of overlapping of coverages. These interferences become trivial when, unlike our assumption in this paper, there are only a few femtocells deployed at a good distance from the macro eNB where the macro coverage is very poor. In this case, the interference management typically does not take up a great challenge and the ordinary interference management techniques, for example, simple FFR schemes or hard frequency reuse (HFR), may be chosen.

In this paper, we investigate the capacity improvement scopes for femtocell systems with FFR deployment. First, we formulate the capacity improvement problem as an optimization problem, whose objective is to maximize the throughput with the constraints of transmit power limits. The complexity of the problem calls for sub-optimal solutions. We propose a sub-optimal solution and simulation results show that the proposed scheme attains higher capacity or throughput compared to the existing schemes.

The remainder of this paper is organized as follows. Commonly known FFR deployment schemes are described briefly in Sec. 2. In Sec. 3, we introduce the system model and formulate the problem. In Sec. 4, we present some considerations aiming to reach a sub-optimal solution to the problem. Then we propose a practical sub-optimal solution in Sec. 5. Simulation results are given in Sec. 6 and the whole paper is concluded in Sec. 7.

\section{Common FFR Schemes in Femtocell Systems}

The commonly known schemes for FFR, while considering deployment in femtocell systems, are briefly described in this section.

\subsection{Strict FFR}

A cell is partitioned into two regions in this scheme: the center zone and the edge zone. For a cluster of $N$ cells, the frequency band is divided into $(N+1)$ sub-bands. The center zones of all cells are allocated to a common subband. The rest of the sub-bands are allocated to the edge zone of the cells depending on the frequency reuse factor (FRF). Here, FRF is 1 and $N$ for the center zone and the edge zone, respectively. A HeNB in the center zone uses the sub-band that is used by the eNB in the edge zone. Similarly, a Home eNB in the edge zone uses the sub-band that is used by the eNB in the center zone. [15] shows that the throughput maximizes when the center zone radius is set to 0.65 times the macrocell radius.

\subsection{Soft FFR}

A cell is partitioned into center zone and edge zone. For a cluster of $N$ cells, the frequency band is divided into $N$ sub-bands. The edge zones of the cells in a cluster are allocated to different sub-bands and the center zone uses the sub-band selected for the neighboring cell's edge zone. Similar to strict FFR, a HeNB in the center zone uses the sub-band that is used by the eNB in the edge zone and a HeNB in the edge zone uses the sub-band that is used by the eNB in the center zone.

\subsection{FFR-3}

A cell is partitioned into center zone and edge zone along with three sectors. The frequency band is divided into four sub-bands, namely, A, B, C and D where subband A is made larger than others. Sub-band A is allocated to the center zone and the remaining three sub-bands are allocated to the edge zone of the three sectors. Here, FRF is 1 and 3 for the center zone and the edge zone, respectively. A HeNB in the center zone uses all the sub-bands except the sub-band that is used by the eNB in the center zone and a HeNB in the edge zone uses all the sub-bands except the one that is used by the eNB in that particular zone. [11] shows that the throughput maximizes when the center zone radius is set to 61 percent of the macrocell radius.

\subsection{Optimal Static FFR (OSFFR)}

[11] proposes OSFFR. Here, a cell is partitioned into center zone and edge zone along with six sectors. The frequency band is divided into seven sub-bands where one of the sub-bands is made larger than others and it is allocated to the center zone. The remaining six sub-bands are allocated to the edge zone of the six sectors. Here, FRF is 1 and 6 for the center zone and the edge zone, respectively. A HeNB in the center zone uses all sub-bands, except the one used in the center zone by the eNB as well as the subbands used by the eNB in the edge zone of the same sector and in the two edge zones adjacent to that sector. A HeNB in the edge zone uses all sub-bands, except the sub-band used by the eNB in the same edge zone. [11] shows that the throughput maximizes when the center zone radius is set to 54 percent of the macrocell radius.

\subsection{3-Layer/3-Sector FFR}

[12] proposes this scheme. Here, a cell is partitioned into three zones: inner or center zone, intermediate zone and outer zone along with three sectors. The frequency band is divided into four sub-bands. One sub-band is allocated to the center zone. The rest three sub-bands are allocated to the intermediate zones with FRF 3. Similarly, these three sub-bands are also allocated to the outer zones with FRF 3. However, the same two sub-bands are not allocated to the intermediate zone and outer zone of the same sector. A HeNB, whether it is in the center zone, 
intermediate zone or outer zone, uses all sub-bands except the one used by the eNB in its own zone.

\section{System Model and Problem Formulation}

We consider a hexagonal multi-cell LTE femtocell based network with FFR deployment. We consider sectorization of the cells. We assume that the sectors in a cell are sequentially numbered clockwise around the cell and represented as $S$ and there are $S$ numbers of sectors in each cell. In addition, there can be different regions based on the distance from the macro eNB in each sector. This kind of regions in each sector will be termed as layers in this paper. We assume that the layers in a sector are represented as $l$ where $l=1$ represents the center layer. $l$ sequentially increases outwards and there are $L$ numbers of layers in each sector. We assume that the sub-carriers are represented as $k$ and there are $K$ numbers of sub-carriers in the whole available bandwidth. The downlink SINR for macro-user $m$ on sub-carrier $k$ can be expressed by

$$
\operatorname{SINR}_{m}^{k}=\frac{P_{M}^{k} G_{m, M}^{k}}{N_{0} \Delta f+\sum_{M^{\prime} \neq M} P_{M^{\prime}}^{k} G_{m, M^{\prime}}^{k}+\sum_{F} P_{F}^{k} G_{m, F}^{k}}
$$

where $G_{m, M}^{k}$ and $G_{m, M}^{k}$, represent the channel gain, on subcarrier $k$, between macro-user $m$ and serving macrocell $M$ and between user $m$ and neighbor macrocells $M^{\prime}$, respectively. $P^{k}{ }_{M}$ and $P^{k}{ }_{M}$, are the transmit power on sub-carrier $k$, of serving cell $M$ and whole neighbor cell set $M^{\prime}$, respectively. $P_{F}^{k}$ is the transmit power of neighbor femtocells $F$ on sub-carrier $k . G_{m, F}^{k}$ represents the channel gain between user $m$ and neighbor femtocells on sub-carrier $k$. $N_{0}$ is the white noise power spectral density, and $\Delta f$ refers to the subcarrier spacing. $\Delta f$ is set to $15 \mathrm{kHz}$ in LTE.

Similarly, the downlink SINR for femto-user $f$ on subcarrier $k$ can be expressed by

$$
\operatorname{SINR}_{f}^{k}=\frac{P_{F}^{k} G_{f, F}^{k}}{N_{0} \Delta f+\sum_{M} P_{M}^{k} G_{f, M}^{k}+\sum_{F^{\prime} \neq F} P_{F^{\prime}}^{k} G_{f, F^{\prime}}^{k}}
$$

where $G_{f, F}^{k}$ and $G_{f, F}^{k}$, represent the channel gain, on subcarrier $k$, between femto-user $f$ and serving femtocell $F$ and between user $f$ and neighbor femtocells $F^{\prime}$, respectively. $P_{F}^{k}$ and $P_{F}^{k}$, are the transmit power on sub-carrier $k$, of serving cell $F$ and whole neighbor cell set $F^{\prime}$, respectively. $P^{k}{ }_{M}$ is the transmit power of neighbor macrocells $M$ on sub-carrier $k$. $G_{f, M}^{k}$ represents the channel gain between user $f$ and neighbor macrocells on sub-carrier $k$.

A macro- or femto-user is represented as $u$ where $u \in\{m, f\}$ The channel gain is dominantly affected by Rayleigh fading $H$, log-normal shadowing $X_{\alpha}$, and path losses $P L_{u}$. So, the channel gain of a user $u$ on sub-carrier $k$ can be shown as follows [16]:

$$
G_{u, M \text { orF }}^{k}=10^{\left(-P L_{u}+X_{\alpha}\right) / 10} \cdot\left|H_{u}^{k}\right|^{2} .
$$

Path loss is calculated according to two formulas, shown in Tab. 2 [10]. The practical capacity of a user $u$ on sub-carrier $k$ can be given by

$$
C_{u}^{k}=\Delta f \cdot \log _{2}\left(1+\alpha \operatorname{SINR} R_{u}^{k}\right)
$$

where $\alpha$ a is a constant for target Bit Error Rate (BER), and defined by $\alpha=-1.5 / \ln (5 B E R)$ [16]. Here, we set BER to $10^{-6}$.

We assume that there are $N_{m}^{S, l}$ and $N_{f}^{S, l}$ number of macro- and femto-users in layer $l$ of sector $s$, respectively. The overall cell throughput for macro-users can be expressed as follows:

$$
R_{M}=\sum_{s=1}^{S} \sum_{l=1}^{L} \sum_{m=1}^{N_{m}^{s, l}} \sum_{k=1}^{K} \beta_{m}^{k} C_{m}^{k}
$$

where $\beta_{m}{ }^{k}$ represents the sub-carrier assignment to macrousers. When the sub-carrier $k$ is assigned to macro user $m$, $\beta_{m}{ }^{k}=1$ and otherwise, $\beta_{m}{ }^{k}=0$. Thus,

$$
\beta_{m}^{k} \in\{0,1\}, \forall m \text {. }
$$

Similarly, the overall cell throughput for femto-users can be expressed as follows:

$$
R_{F}=\sum_{s=1}^{S} \sum_{l=1}^{L} \sum_{f=1}^{N_{f}^{s, l}} \sum_{k=1}^{K} \beta_{f}^{k} C_{m}^{k}
$$

where $\beta_{f}^{k}$ represents the sub-carrier assignment to femtousers. When the sub-carrier $k$ is assigned to femto-user $f$, $\beta_{f}^{k}=1$, and otherwise, $\beta_{f}^{k}=0$. Thus,

$$
\beta_{f}^{k} \in\{0,1\}, \forall f .
$$

The total transmit power of a macro eNB $P_{e N B}$ cannot exceed its maximum transmit power capability $P_{e N B}^{\max }$. So,

$$
P_{e N B}=\sum_{s=1}^{S} \sum_{l=1}^{L} \sum_{m=1}^{N_{m}^{s, l}} \sum_{k=1}^{K} \beta_{m}^{k} P_{M}^{k} \leq P_{e N B}^{\max } .
$$

Similarly, the total transmit power of a HeNB $P_{H e N B}$ cannot exceed its maximum transmit power capability $P_{H e N B}^{\max }$. Assuming that a Home eNodeB serves a total of $N_{f}^{F}$ number of femto-users,

$$
P_{H e N B}=\sum_{f=1}^{N_{f}^{F}} \sum_{k=1}^{K} \beta_{f}^{k} P_{F}^{k} \leq P_{H e N B}^{\max } .
$$

The total cell throughput is $R_{T}=R_{M}+R_{F}$. So, the optimization problem can be formulated as follows while satisfying (9) and (10):

$$
\begin{array}{r}
\max _{S, L, \beta_{m}^{k}, \beta_{f}^{k}} R_{T}={ }_{S, L, \beta_{m}^{k}, \beta_{f}^{k}}^{\max } \sum_{s=1}^{S} \sum_{l=1}^{L} \sum_{m=1}^{N_{m}^{s, l}} \sum_{k=1}^{K} \beta_{m}^{k} C_{m}^{k} \\
+\sum_{s=1}^{S} \sum_{l=1}^{L} \sum_{f=1}^{N_{f}^{s, l}} \sum_{k=1}^{K} \beta_{f}^{k} C_{m}^{k}
\end{array}
$$

The optimization problem is NP-complete for which the solution is not evident. FFR schemes described in 
Sec. 2 are essentially few existing or already proposed suboptimal solutions. In this paper, we propose a sub-optimal solution that achieves higher overall throughput identifying the solution closer to the optimum.

\section{Considerations for a Sub-optimal Solution}

We describe below some considerations that aim to reach a sub-optimal solution for use in practice. We assume that the assignment of sub-carrier $k$ in layer $l$ of sector $s$ for macro- and femto-users are represented by $\beta_{m, s, l}^{k}$ and $\beta_{f, s, l}^{k}$, respectively. $\beta_{m, s, l}^{k}$ or $\beta_{f, s, l}^{k}$ are set to 1 , if the sub-carrier $k$ is assigned and set to 0 , otherwise.

i. To limit CCI, a sub-carrier can be allocated to only one macro-user in each sector. So,

$$
\sum_{l=1}^{L} \sum_{m=1}^{N_{m}^{s, l}} \beta_{m}^{k} \in\{0,1\}, \forall k .
$$

ii. To limit cross-tier interference, we assume that the same sub-carriers are not allocated to macro- and femto-users in the same layer in a sector. Thus,

$$
\beta_{m, s, l}^{k} \neq \beta_{f, s, l}^{k} \text { if } \beta_{m, s, l}^{k}=1, \forall k .
$$

iii. The central layer can be allocated sub-carriers using $\mathrm{FFR}=1$. Identifying these sub-carriers as $k_{C}$ and the center layer as $l=C$,

$$
\beta_{m, s, l=C}^{k}=1, \text { and } \beta_{m, s, l \neq C}^{k}=0, \quad k=k_{C} .
$$

Since 1 is the lowest frequency reuse factor, $k_{C}$ may include a good number of sub-carriers from the available bandwidth.

iv. When $k \neq k_{C}$, to limit CCI, we assume that the same sub-carriers are not allocated to macro-users in adjacent sectors in a cell. Since sector $s+1$ and $s-1$ are adjacent to sector $s$,

$$
\begin{aligned}
& \beta_{m, s, l}^{k} \neq \beta_{m, s+1, l}^{k}, \text { if } \beta_{m, s, l}^{k}=1, \quad k \neq k_{C} \\
& \text { and } \beta_{m, s, l}^{k} \neq \beta_{m, s-1, l}^{k}, \text { if } \beta_{m, s, l}^{k}=1, \quad k \neq k_{C} .
\end{aligned}
$$

v. Since the two outer layers of adjacent cells lie next to each other, to limit CCI, we assume that the same sub-carriers are not allocated to macro-users in these outer layers. In a hexagonal multi-cell network, the sector of the adjacent cell, which is aligned with sector $s$, can be equivalently found as $\lfloor s+S / 2\rfloor$. Identifying the outer layers as $l=O$,

$$
\beta_{m, s, l=0}^{k} \neq \beta_{m,\left|s+\frac{s}{2}\right|, l=0}^{k} \text {, if } \beta_{m, s, l=0}^{k}=1, k \neq k_{C}
$$

vi. When $\beta_{f, s, l}^{k}=1$, as $l$ decreases, the associated $P^{k}{ }_{M}$ can be decreased due to smaller path loss and the sub-carrier allocation causes less interference to neighboring cells. Similarly, as $l$ decreases, the associated $G_{m, M}^{k}$ decreases due to larger path loss from the neighbor macrocells and the sub-carrier allocation suffers from less interference.

vii. When $\beta_{m, s, l}^{k}=1$, the associated $\operatorname{SINR}_{m}^{k}$ improves as $l$ decreases and so, the macro service can use the subcarrier more efficiently. On the other hand, when $\beta_{f, s, l}^{k}=1$, the associated $\operatorname{SINR}_{f}^{k}$ improves as $l$ increases because of reduced cross-tier interference and so, the femtocell operation can use the sub-carrier more efficiently.

viii. $\beta_{f, s, l}^{k}=1$ may be used repeatedly for different femtocells together leading to increased femtocell throughput. However, in this case, the HeNBs need to be installed at predesigned places to limit the co-tier interference and the cross-tier interference as well.

ix. A macro- or femto-user can only be allocated one or more whole resource blocks (RBs). Each RB consists of $12 \mathrm{sub}$-carriers and so, it is $180 \mathrm{kHz}$ wide. Let us assume that the $n$ numbered RB contains sub-carriers $\{n 1, n 2, n 3, . ., n x, \ldots, n 12\}$. Thus,

if $\beta_{m, s, l}^{k}=1$ where $k \in n x$, then $\beta_{m, s, l}^{n x}=1, \forall n x$.;

if $\beta_{f, s, l}^{k}=1$ where $k \in n x$, then $\beta_{f, s, l}^{n x}=1, \forall n x$.

$\mathrm{x}$. It is possible to vary the resource share more gradually with distance from the eNB using higher value of $L . L=3$ is physically realizable with tight radio planning and [12] proposes three layers in each sector. However, $L>3$ is formidable and so, it may not be considered.

xi. Higher sectorization uses narrower antenna beamwidth at the eNB and thus, causes less inter-cell interference. So, a high value of $S$ can reduce the inter-cell cross-tier interference. $S=6$ is physically realizable with tight radio planning and [11] considers six sectors in each cell for FFR. However, $S>6$ is formidable and so, it may not be considered.

\section{Proposed Scheme}

In this section, we present a greedy heuristic algorithm, based on the considerations in Sec. 4, for frequency resource allocation with an attempt for a sub-optimal solution with better throughput. For simplicity, we assume uniform distribution of the users. To attain efficient use of sub-carriers, we propose higher resource share for macro use in areas closer to the eNB and also, higher resource share for femto use in areas further away from the eNB. In order to materialize such variation in frequency allocation more gradually, we use high value for $L$ and so, we set $L=3$. [12] already proposes three layers with three sectors in each cell. Besides, to reduce the inter-cell cross-tier interference, our proposal uses six sectors. It is shown that a 6-sector cell, in general, can attain significantly higher cell throughput compared to a 3 -sector cell [17]. The use of $S=6$ along with $L=3$ requires high number of antennas and a lot of cabling [18]. Also, it will engender high num- 
ber of interferers and overlapping regions and so, a very delicate radio planning will be required [17]. However, these difficulties may be accepted due to the improvement in throughput shown in Sec. 6 to meet the high data rate demand.

Identifying the intermediate layers as $l=I$, we set $l \in\{O, I, C\}$. There are a total of 18 sub-areas, namely, O1, $\mathrm{O} 2, \mathrm{O} 3, \mathrm{O} 4, \mathrm{O} 5$ and $\mathrm{O} 6$ in the outer region; I1, I2, I3, I4, I5 and $\mathrm{I} 6$ in the intermediate region; $\mathrm{C} 1, \mathrm{C} 2, \mathrm{C} 3, \mathrm{C} 4, \mathrm{C} 5$ and $\mathrm{C} 6$ in the center region, as shown in Fig. 1. We assume that the outer, intermediate and center regions are identified as $\mathrm{O}_{i}^{m}, \mathrm{I}_{i}^{m}$ and $C_{i}^{m}$, respectively, for macro services in sector $i$. Similarly, the outer, intermediate and center regions are identified as $\mathrm{O}_{i}^{f}, \mathrm{I}_{i}^{f}$ and $C_{i}^{f}$, respectively, for femto services in sector $i$. We propose a resource allocation scheme based on the considerations in Sec. 4 with an attempt to minimize all kinds of interferences. To exemplify the resource allocation scheme, the available bandwidth is divided into 7 sub-bands (SBs), denoted as, A, B, C, D, E, F and G. Each sub-band consists of a number of complete resource blocks (RBs). The sub-bands A to $F$ are sequentially numbered twice from 1 through 6 and also, from 7 through 12 (i.e., both $\mathrm{SB}_{2}$ and $\mathrm{SB}_{8}$ represent sub-band $\mathrm{B}$ ). The sub-band $\mathrm{G}$ is denoted as $\mathrm{SB}_{\mathrm{C}}$. Algorithm 1 shows an example of allocation of sub-bands in different sub-areas for macro and femto services. Figure 1 illustrates this allocation.

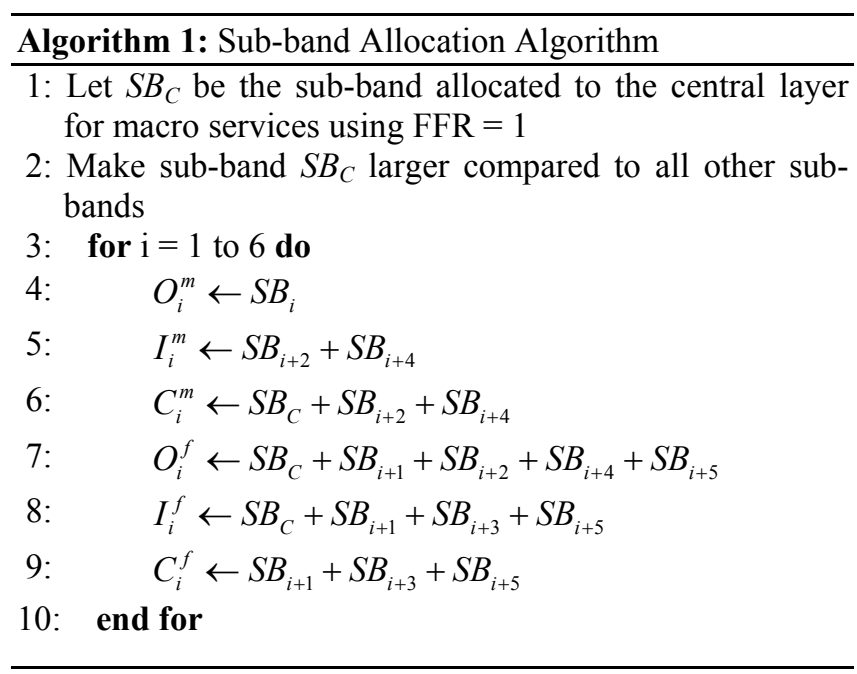

Table 1 demonstrates the relative frequency resource share for macro use as well as femto use in different layers. It is assumed that each of the sub-bands from A to F contains 6 RBs and the sub-band $\mathrm{G}$ contains $14 \mathrm{RBs}$. The radius of center and intermediate layers are chosen 0.4 and 0.7 times the macrocell radius, respectively. For macro services, when the same sub-band is used in adjacent layers, the sub-band is assumed to be uniformly used over the whole area and the frequency resource used per unit area is computed to determine the resource share in each layer. The cell is considered circular in this computation. For femto services, the relative amount of sub-band allocation in different layers is used to compute the relative resource share. As Table 1 shows, there is an achievement of ample gradual decrease and increase in resource share with dis- tance from the eNB for macro use and femto use, respectively, leading to an efficient utilization of the resources.

The sub-band sizes may vary and we suggest a careful selection of sub-band sizes considering potential data rate requirements. Our proposal limits itself to a fixed resource allocation based on the available radio network planning (RNP) related information. It obviates the need for adaptive control. However, an extension of the proposal can be adaptive allocation considering the channel quality of the users at different frequencies as well as their current data rate demands. In this case, the resource share is adjusted with time among eNodeBs, sectors and layers. The channel-dependent scheduling (CDS) will be more efficient with the adaptive allocation. The eNodeBs may use a distributive control of adaptive resource allocation with an attempt for local optimization. For this purpose, new fields can be added to the Load Information message, which currently exists in the specification for exchange of information between neighboring eNodeBs over the X2 interface. These fields may contain information like the estimated interference at different frequencies, channel quality of the users at different frequencies and current load demand in each layer of each sector of the macrocells. It is possible to support the additional overhead in Load Information message because X2 can be a high speed link.

\begin{tabular}{|c|c|c|}
\hline \multirow{2}{*}{ Layer } & \multicolumn{2}{|c|}{ Relative Resource Share } \\
\cline { 2 - 3 } & Macrocell & Femtocell \\
\hline Center & 3.321 & 0.474 \\
\hline Intermediate & 2.082 & 0.842 \\
\hline Outer & 1 & 1 \\
\hline
\end{tabular}

Tab. 1. Relative resource share in different layers.

The proposed scheme could further improve its performance if it was possible to transfer some macro-users to femto service in cell edge areas. Therefore, we suggest that the operation of HeNBs in open access mode can be motivated in outer layers and there, a large number of HeNBs can be installed. This can allow some cell edge users to be served by HeNBs instead of the macro eNB and receive better throughput provided that they are located close to any HeNB.

\section{Simulation}

The proposed method was simulated using parameter values shown in Tab. 2. It used Monte Carlo simulation with one thousand iterations. It considers a one-tier LTE network. Both the users and the femtocells are distributed randomly in each layer of each sector of the macrocells. The number of femtocells was varied while evaluating the throughput using equations (1)-(7). Simulation was also performed for existing schemes, namely, FFR-3, OSFFR and 3-layer/3-sector FFR and these schemes were compared with the proposed scheme. Figure 2 shows that the macro throughput of the proposed scheme is significantly higher than the existing schemes. In general, the macro service is considered more important compared to the femto service and also, the macro service is usually the 


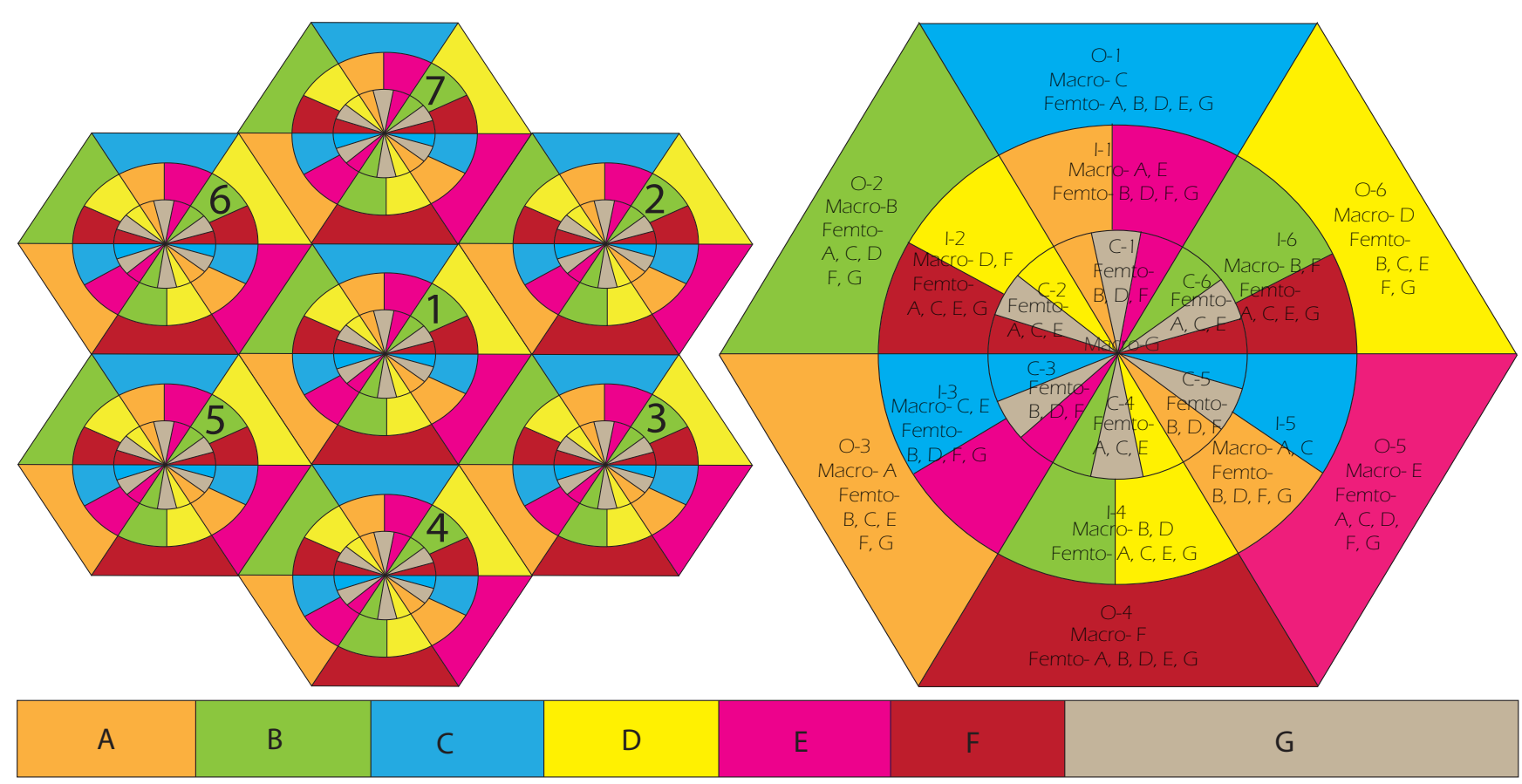

Fig. 1. FFR deployment in proposed scheme.

\begin{tabular}{|c|c|c|}
\hline Parameter & Macrocell & Femtocell \\
\hline Number of cells & 7 & 66 \\
\hline Cell radius & $280 \mathrm{~m}$ & $30 \mathrm{~m}$ \\
\hline eNB Transmit Power & $22 \mathrm{~W}, 19 \mathrm{~W}, 15 \mathrm{~W}$ & $20 \mathrm{~mW}$ \\
\hline No. of users in a cell & 50 & 1 \\
\hline Channel Bandwidth & \multicolumn{2}{|c|}{$10 \mathrm{MHz}$} \\
\hline No. of RBs & \multicolumn{2}{|c|}{50} \\
\hline Sub-band size $(\mathrm{A}-\mathrm{F})$ & \multicolumn{2}{|c|}{$1.08 \mathrm{MHz}(6 \mathrm{RBs})$} \\
\hline Sub-band size $(\mathrm{G})$ & \multicolumn{2}{|c|}{$2.52 \mathrm{MHz}(14 \mathrm{RBs})$} \\
\hline White noise power density & \multicolumn{2}{|c|}{$-174 \mathrm{dBm} / \mathrm{Hz}$} \\
\hline Center layer radius & \multicolumn{2}{|c|}{0.4 of macrocell radius } \\
\hline Intermediate layer radius & \multicolumn{2}{|c|}{0.7 of macrocell radius } \\
\hline $\begin{array}{l}\text { Channel model: path loss } \\
\text { (outdoor) }\end{array}$ & \multicolumn{2}{|c|}{$28+35 \log 10(d) \mathrm{dB}$} \\
\hline $\begin{array}{l}\text { Channel model: path loss } \\
\text { (indoor) }\end{array}$ & \multicolumn{2}{|c|}{$\begin{array}{c}P L=38.5+20 \log 10(d)+7 \mathrm{~dB}, \quad 0<d \leq 10 \\
P L=38.5+20 \log 10(d)+10 \mathrm{~dB}, 10<d \leq 20 \\
P L=38.5+20 \log 10(d)+15 \mathrm{~dB}, 20<d \leq 30\end{array}$} \\
\hline
\end{tabular}

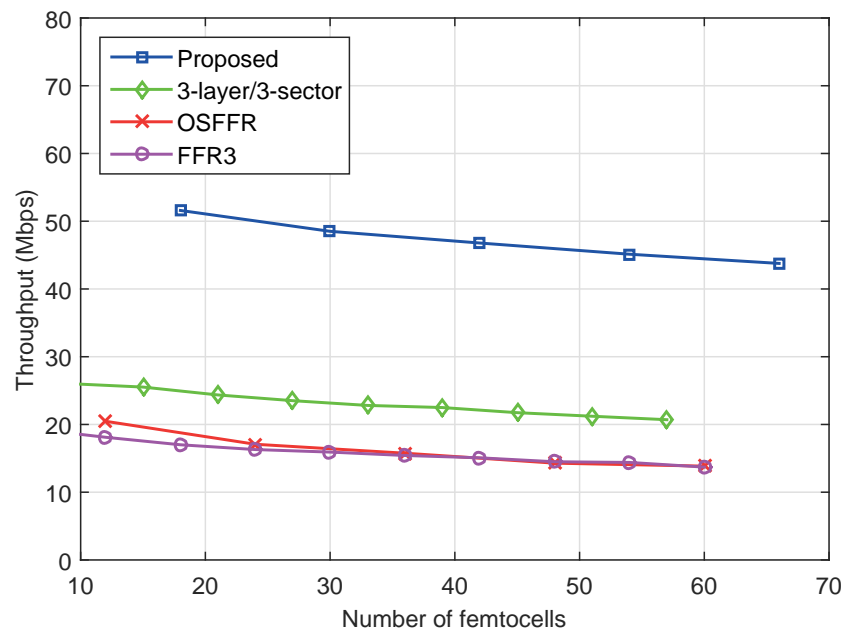

Fig. 2. Macro throughput vs. number of femtocells.

Tab. 2. Simulation assumptions.

major source of revenue for the operator. So, the achievement of the proposed scheme in macro throughput is a conspicuous success. As the number of femtocell is increased, the macro throughput tends to decrease because the macro-users receive higher interference from the femtocells.

Figure 3 shows that the femtocell throughput increases with the number of femtocells. However, the growth of the femtocell throughput is fairly impaired primarily because of the co-tier interference and so, the impairment increases with the number of femtocells. The femtocell throughput of the proposed scheme is little higher than FFR-3 and 3-layer/3-sector FFR but it is lower than

OSFFR. OSFFR offers much better femtocell throughput because OSFFR, with its two layers, yields less interference to the femtocells. Figure 4 shows the comparison of the overall throughput, which is the summation of throughput of macro and femto cells. Evidently, the overall throughput of the proposed scheme is considerably higher than the existing schemes. Figure 5 shows macro throughput only in outer and intermediate layers for the proposed scheme and 3-layer/3-sector FFR to demonstrate the individual improvement in those layers.

The simulation results markedly show that the proposed scheme is more attractive than the existing schemes in terms of the throughput or the spectral efficiency or the capacity of the system. 


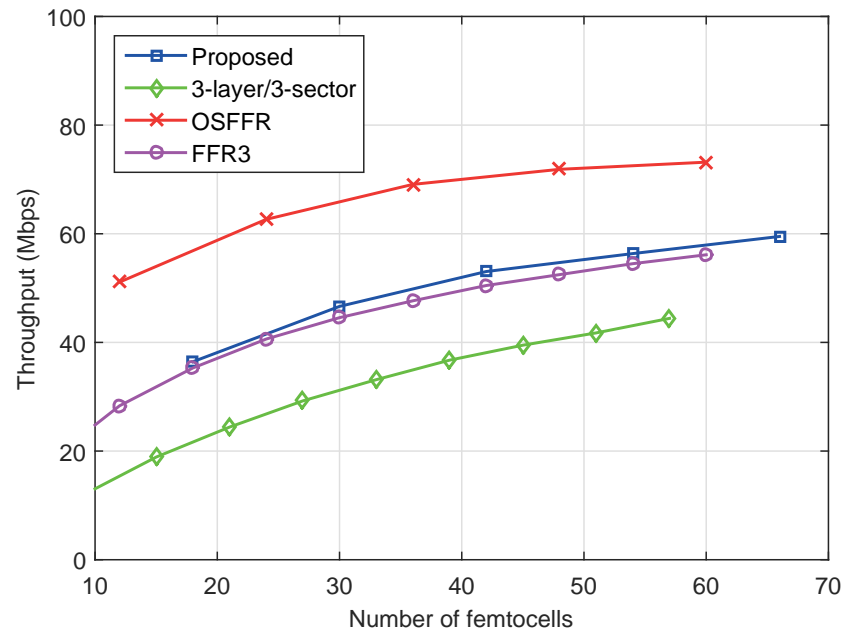

Fig. 3. Femto throughput vs. number of femtocells.

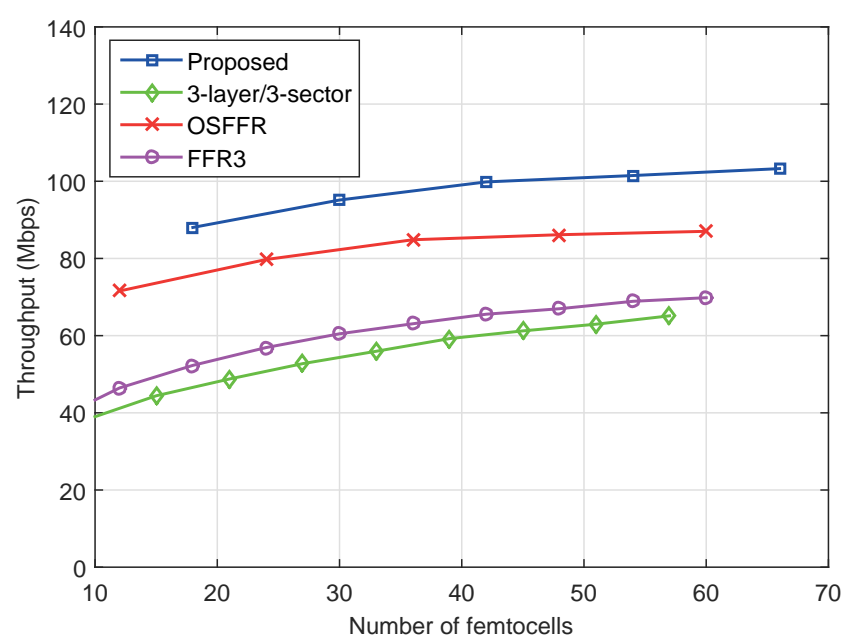

Fig. 4. Overall throughput vs. number of femtocells.

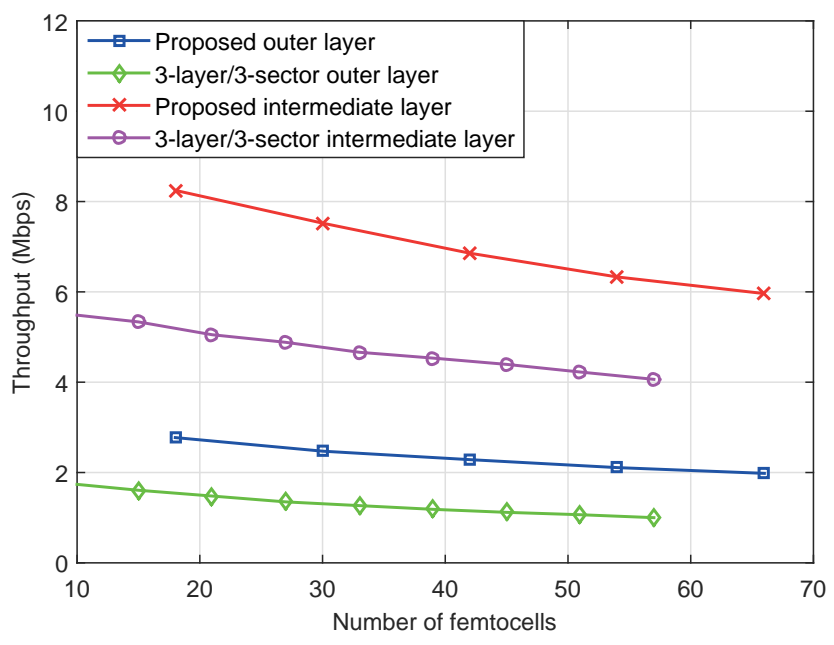

Fig. 5. Macro throughput in outer and intermediate layers vs. number of femtocells.

\section{Conclusion}

The proposed resource allocation scheme uses effective variation in resource share for macrocells and femto- cells with distance and satisfactory interference mitigation applying high number of layering and sectoring. It achieves throughput significantly higher than the existing schemes. It requires high number of antennas, a lot of cabling and a very delicate radio planning. But these downsides can be overshadowed by the fact that the achievement of high throughput or spectral efficiency is of paramount importance to meet the present and future data rate demand.

\section{References}

[1] KAWSER, M. T. LTE Air Interface Protocols. Artech House, 2011, ISBN: 978-1-60807-201-9

[2] BENDLIN, R., CHANDRASEKHAR, V., CHEN, R., et al. From homogeneous to heterogeneous networks: A 3GPP Long Term Evolution. Rel. 8/9 case study. In Proc. of $45^{\text {th }}$ Annual Conference on Information Sciences and Systems (CISS). Baltimore (MD, USA), Mar. 2011, 5 p. DOI: 10.1109/CISS.2011.5766247

[3] SHI, Y., MACKENZIE, A. B., DASILVA, L. A, et al. On resource reuse for cellular networks with femto and macrocell coexistence. In Proc. of the IEEE Global Telecommunication Conference (Globecom). Miami (FL, USA), Dec. 2010, 6 p. DOI: 10.1109/GLOCOM.2010.5683443

[4] FUJITSU NETWORK COMMUNICATIONS. 4G Femtocell for the Dense Metropolitan Environment, 2013. Available at: http://www.fujitsu.com/us/Images/4G-Femtocell-for-DenseMetro-Environment.pdf

[5] NECKER, M. C. Local interference coordination in cellular OFDMA networks. In IEEE 66th Vehicular Technology Conference (VTC 2007 Fall). Baltimore (MD, USA), Sept. 2007, p. 1741-1746. DOI: 10.1109/VETECF.2007.368

[6] LEI, H., ZHANG, L., ZHANG, X, et al. A novel multi-cell OFDMA system structure using fractional frequency reuse. In IEEE 18th International Symposium on Personal, Indoor and Mobile Radio Communications (PIMRC). Athens (Greece), Sept. 2007, 5 p. DOI: 10.1109/PIMRC.2007.4394228

[7] ASSAAD, M. Optimal Fractional Frequency Reuse (FFR) in multicellular OFDMA system. In IEEE Vehicular Technology Conference (VTC 2008 Fall). Calgary (BC), Sept. 2008, 5 p. DOI: 10.1109/VETECF.2008.381

[8] HASSAN, N. U. L., ASSAAD, M. Optimal fractional frequency reuse (FFR) and resource allocation in multiuser OFDMA system. In Proceedings of International Conference on Information and Communication Technologies (ICICT 2009). Karachi (Pakistan), Aug. 2009, p. 88-92. DOI: 10.1109/ICICT.2009.5267207

[9] 3GPP, R1-050507, Huawei. Soft frequency reuse scheme for LTE, 2005.

[10] LEE, P., LEE, T., JEONG, J. et al. Interference management in LTE femtocell systems using fractional frequency reuse. In Proc. of 12th International Conference on Advanced Communication Technology (ICACT). Phoenix Park, Feb. 2010, vol. 2, p. 1047 to 1051. ISBN: 978-1-4244-5427-3

[11] SAQUIB, N., HOSSAIN, E., KIM, D. I. Fractional frequency reuse for interference management in LTE-advanced HetNets. IEEE Wireless Communications, April 2013, vol. 20, no. 2, p. 113-122. DOI: 10.1109/MWC.2013.6507402

[12] FRADI, N., NAJEH, S., BOUJEMAA, H. Resource allocation in OFDMA networks with femto and macro-cells coexistence using Fractional Frequency Reuse (FFR). In International Conference on Communications and Networking (ComNet). Hammamet (Tunisia), March, 2014, 5 p. DOI: 10.1109/COMNET.2014.6840931

[13] CHANG, R. Y., TAO, Z., ZHANG, J., et al. A graph approach to dynamic fractional frequency reuse (FFR) in multi-cell OFDMA 
networks. In Proceedings of IEEE International Conference on Communications (ICC 2009). Dresden (Germany), June 2009, 6 p. DOI: 10.1109/ICC.2009.5198612

[14] FANG, L., ZHANG, X. Optimal fractional frequency reuse in OFDMA based wireless networks. In Proceedings of 4th International Conference on Wireless Communications, Networking and Mobile Computing, (WiCOM'08). Dalian (China), 4 p. DOI: 10.1109/WICOM.2008.166

[15] NOVLAN, T., ANDREWS, J. G., ILlSOO SOHN, et al. Comparison of fractional frequency reuse approaches in the OFDMA cellular downlink. In Proc. of the IEEE Global Telecommunication Conference GLOBECOM 2010. Miami (FL, USA), Dec. 2010, 5 p. DOI: 10.1109/GLOCOM.2010.5683973

[16] LEE, T., YOON, J., LEE, S., et al. Resource allocation analysis in OFDMA femtocells using fractional frequency reuse. In IEEE $21^{s t}$ International Symposium on Personal, Indoor and Mobile Radio Communication (PIMRC). Istanbul (Turkey), 2010, p. 1224-1229. DOI: 10.1109/PIMRC.2010.5672042

[17] KUMAR, S., KOVACS, I. Z., MONGHAL, G., et al. Performance evaluation of a 6-sector-site deployment for downlink UTRAN long term evolution. In IEEE Vehicular Technology Conference (VTC 2008 Fall). Calgary (BC), Sept. 2008, 5 p. DOI: 10.1109/VETECF.2008.384

[18] SCANFERLA, D. Studies on 6-Sector-Site Deployment in Downlink LTE. Technische Universiteit Eindhoven (TUE), 2012. ISBN: 978-90-444-1128-7

\section{About the Authors ...}

Mohammad T. KAWSER is an Assistant Professor at the Electrical and Electronic Engineering Department at
Islamic University of Technology, Bangladesh. His work experience includes Qualcomm, Inc., CA, USA and Accuver Americas (formerly, WirelessLogix, Inc), TX, USA. He received MS degree from Virginia Tech, USA in 2005 and BS degree from Bangladesh University of Engineering and Technology, Bangladesh in 1999, both in Electrical Engineering. His current research interests include layer 2 and layer 3 functions of cellular operation, LTE-Advanced features, etc.

Mohammad R. ISLAM received BS and MS degree in Electrical and Electronic Engineering from Bangladesh University of Engineering and Technology, Bangladesh in 1998 and 2004, respectively. He received MBA degree from the Institute of Business Administration, Bangladesh in 2006. He received his $\mathrm{PhD}$ degree from Kyung Hee University, South Korea in 2010 . He is serving as a Professor at Islamic University of Technology, Bangladesh. His research interests include cooperative technique for wireless sensor networks, LDPC and QC-LDPC codes, secrecy capacity and other wireless applications.

Kazi I. AHMED, Mir R. KARIM and Jaeed B. SAIF are currently senior level students in BS program in Electrical and Electronic Engineering at Islamic University of Technology, Bangladesh and they are expected to graduate soon. They all have been awarded the full OIC scholarship. Their research interests include interference management, resource allocation in femtocell networks and device to device communications. 